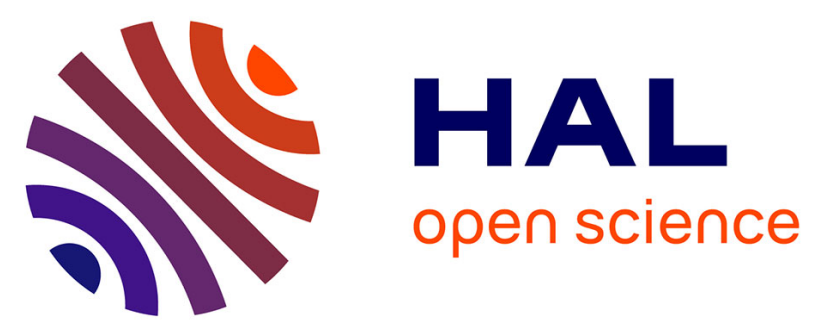

\title{
Towards a patient-specific hepatic arterial modeling for microspheres distribution optimization in SIRT protocol
}

Costanza Simoncini, Krzysztof Jurczuk, Daniel Reska, Simon Esneault, Jean-Claude Nunes, Jean-Jacques Bellanger, Hervé Saint-Jalmes, Yan Rolland, Pierre-Antoine Eliat, Johanne Bezy-Wendling, et al.

\section{To cite this version:}

Costanza Simoncini, Krzysztof Jurczuk, Daniel Reska, Simon Esneault, Jean-Claude Nunes, et al.. Towards a patient-specific hepatic arterial modeling for microspheres distribution optimization in SIRT protocol. Medical and Biological Engineering and Computing, 2018, 56 (3), pp.515-529. 10.1007/s11517-017-1703-1 . hal-01740212

\section{HAL Id: hal-01740212 \\ https://hal-univ-rennes1.archives-ouvertes.fr/hal-01740212}

Submitted on 13 Apr 2018

HAL is a multi-disciplinary open access archive for the deposit and dissemination of scientific research documents, whether they are published or not. The documents may come from teaching and research institutions in France or abroad, or from public or private research centers.
L'archive ouverte pluridisciplinaire HAL, est destinée au dépôt et à la diffusion de documents scientifiques de niveau recherche, publiés ou non, émanant des établissements d'enseignement et de recherche français ou étrangers, des laboratoires publics ou privés. 


\title{
Towards a patient-specific hepatic arterial modeling for microspheres distribution optimization in SIRT protocol
}

\author{
Costanza Simoncini ${ }^{1,2}$. Krzysztof Jurczuk ${ }^{3}$. Daniel Reska ${ }^{3}$. \\ Simon Esneault ${ }^{4}$. Jean-Claude Nunes ${ }^{1,2}$. Jean-Jacques Bellanger ${ }^{1,2}$. \\ Hervé Saint-Jalmes ${ }^{1,2,5}$. Yan Rolland ${ }^{1,2,5}$. Pierre-Antoine Eliat ${ }^{6,7,8}$. \\ Johanne Bézy-Wendling', ${ }^{1,2}$. Marek Kretowski ${ }^{3}$
}

\begin{abstract}
Selective Internal Radiation Therapy (SIRT) using Yttrium-90 loaded glass microspheres injected in the hepatic artery is an emerging, minimally invasive therapy of liver cancer. A personalized intervention can lead to high concentration dose in the tumor, while sparing the surrounding parenchyma. We propose a computational model for patient-specific simulation of entire hepatic arterial tree, based on liver, tumors and arteries segmentation on patient's tomography. Segmentation of hepatic arteries down to a diameter of 0.5 $\mathrm{mm}$ is semi-automatically performed on 3D Cone-Beam CT Angiography. Liver and tumors are extracted from CT-scan at portal phase by an active surface method. Once the images are registered through an automatic multimodal registration, extracted data are used to initialize a numerical model simulating liver vascular net-
\end{abstract}

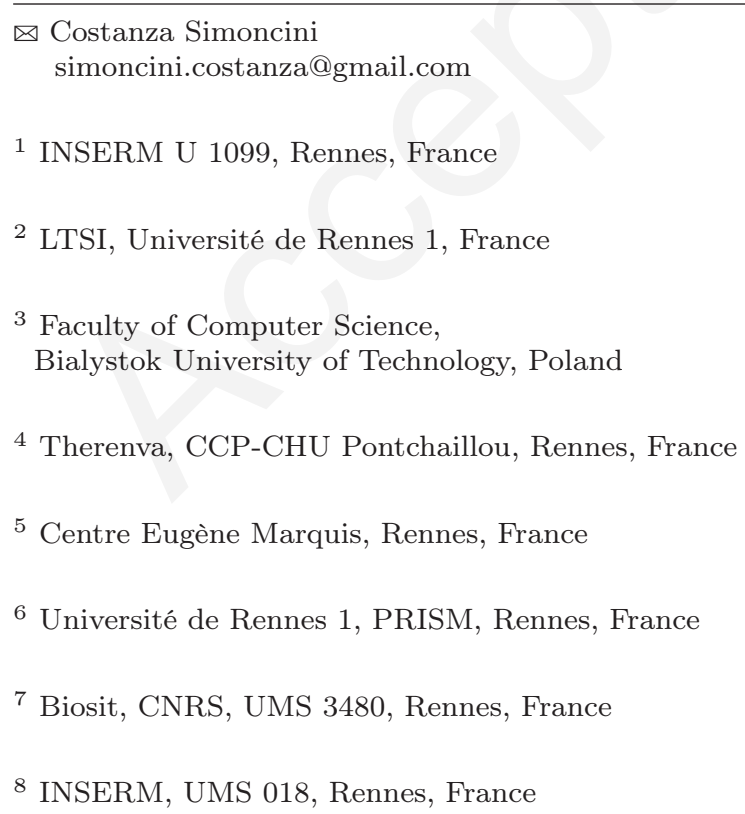

8 INSERM, UMS 018, Rennes, France

work. The model creates successive bifurcations from given principal vessels, observing Poiseuille's and matter conservation laws. Simulations provide a coherent reconstruction of global hepatic arterial tree until vessel diameter of $0.05 \mathrm{~mm}$. Microspheres distribution under simple hypotheses is also quantified, depending on injection site. The patient-specific character of this model may allow a personalized numerical approximation of microspheres final distribution, opening the way to clinical optimization of catheter placement for tumor targeting.

Keywords Computational modeling · hepatic artery • image processing $\cdot$ liver tumor $\cdot$ radioembolization

\section{Introduction}

The Hepato-Cellular Carcinoma (HCC) is the leading cause of primary liver malignancy throughout the world and is the fourth cause of mortality, with more than 500000 deaths per year [10]. Treatment options are limited [1]; a tumor in an early stage can be treated by liver transplantation, resection or percutaneous ablation. Intermediate or advanced stage HCC are treated with chemotherapy. Radiotherapy could be administered as an adjuvant at any stage of an HCC. It is estimated that less than $15 \%$ of patients are candidates for surgery, $50 \%$ for non-surgical treatments and $35 \%$ for best supportive care [1]. The most limiting factors for chemotherapy are the development of drug resistance and its serious side effects [16]; for radiotherapy, it is the risk of causing radiation-induced liver disease [11]. This is why it is important to develop local and regional treatments. The treatment we are interested in is a particular kind of radiotherapy, called Selective Internal Radiation Therapy (SIRT) [11]. It consists in 
the injection of millions of microspheres containing radioactive Yttrium-90, directly into the hepatic artery, through a catheter. Microspheres flow into the liver tumor via its own vascular network and get permanently lodged in its small vessels. Consequently, there is preferential delivery of microspheres to the tumor capillary bed, allowing for higher doses of radiation to be delivered to the tumor, relative to the surrounding nontumor parenchyma.

Even if globally more efficient than other HCC therapies, this methodology still needs to be optimized in terms of time and cost, as well as personalization [10]. A numerical model of vessels and blood flow would help the physician in the choice of the best injection site and of the dose amount, by letting him test different scenarios and optimize tumor targeting. If the numerical model is accurate enough, the distribution of the microspheres in the liver can be predicted, and invasive clinical examinations can be avoided. In fact, microspheres distribution is currently predicted by the injection of an equivalent amount of ${ }^{99 \mathrm{~m}} \mathrm{Tc}$-loaded proteins, whose behavior is however sometimes different from the one of microspheres. Blood and microspheres flow simulation have already been implemented on arteries geometries made of 3-5 bifurcations [5], [4]. Knowing the geometry of the entire arterial tree of the patient seems to be necessary, and angiographic imagery can provide this information only at a limited resolution level. Typically, we can expect to image vessels with a diameter larger than $0.5 \mathrm{~mm}$. Without a realistic simulation of the entire tree, we could not address the problem of the computation of the particles distribution on the existing set of terminal branches of the patient's vasculature. The goal of this work is to develop a model of the complete hepatic arterial vasculature, the most consistent with the patient's liver characteristics down to small capillaries, at least from a statistical point of view.

In section 2.1 we will present the model used to simulate the patient-specific hepatic arterial network, to which we will refer as the "vascular model". This model, extensively described in [15], can simulate the growth of the three liver vascular trees, based on a liver shape and on some initial vessels. In the present work, initialization of the vascular model is done with the segmented patient-specific data. Extraction of quantified data from the medical images is presented in section 2.2. Since the images are acquired through different techniques, an important registration process is necessary to make the images compatible and to correctly initialize the model with the liver envelope and the main vessels. Section 3 presents the results of the segmentation and registration of hepatic arteries, liver and tumors, as well as the global arterial tree obtained through the initial- ization of the vascular model with patient-specific data. We will also present a preliminary simplified simulation of microspheres distribution in the simulated arterial tree and quantification of their deposition in the tissues. Section 4 shows the clinical applications and the future work needed before the physicians could actually implement it in their protocol: a numerical model of the geometry of the vessels is needed for a complete and realistic simulation and analysis of the blood flow inside the liver. This, in turn, permits a more accurate simulation of microspheres transport by blood and of their trajectories through the vessels. The estimation of microspheres distribution in liver is crucial for the administration of SIRT. We will present the principal conclusions of this work in section 5 .

\subsection{Clinical context}

Liver is the largest internal organ of the human body and its role is crucial for metabolism. Blood is supplied by two different paths: portal vein furnishes around $75 \%$ of it, while the remaining $25 \%$ is provided by the hepatic artery, which brings oxygenated blood directly from the aorta [7].

A particularity of $\mathrm{HCC}$ is that it is almost exclusively vascularized by the hepatic artery. This characteristic allows the physician to reach the tumor with a catheter inserted into the femoral artery, guided through the aorta and then directly into the hepatic artery. Moreover, a tumor stimulates the generation of vessels to have access to nutrients. This angiogenic process is pathological, and it results in a disordered and dense network with a high fractal dimension [16]. This arterial hypervascularization of $\mathrm{HCC}$ is the reason why the arterial blood flow within the tumor is 3 to 7 times greater than in the surrounding noncancerous tissue. This means that it is legitimate to hope that some therapeutic agent injected in the arterial tree will be more concentrated in the tumor than in the healthy tissue. This leads the way to a variety of therapies that have improved during the last decades: injection of chemotherapeutic agents, radio-labeled particles, or even non-reactive particles, with the only aim of embolization.

The gold standard for treating intermediate stage HCC is now considered Trans Arterial Chemo Embolization (TACE) [22]. This approach appears to confer some palliative benefit to many patients, but post embolization syndrome can be severe after TACE.

An alternative is represented by Trans-Arterial Radio Embolization (TARE), which is a way to deliver selectively high radiation dose in the area of interest of the liver with far less embolizing characteristics than 
TACE [1]. SIRT falls within this framework. The most frequently reported agents available for TARE are ${ }^{131} \mathrm{I}$ radio-labelled lipiodol and Yttrium-90 loaded microspheres [1]. The first one is strongly limited by the emission of high-energy gamma radiation. The latter was developed recently to avoid radio-protection problems, Yttrium-90 being a pure beta rays emitter [19]. Globally, TARE gives equivalent results than TACE for intermediate stage $\mathrm{HCC}$ and it presents fewer side effects. For end-stage HCC, TARE therapies are found to have even a better outcome than TACE [1].

SIRT can be delivered through two different kinds of microspheres: SIR-Spheres ${ }^{\circledR}$ (SIRTeX Medical Limited, Australia) and Theraspheres ${ }^{\circledR}$ (BTG, UK). Our interest concerns Therasphere ${ }^{\circledR}$, which have a smaller diameter, around $25 \mu \mathrm{m}$, and are made of glass. Their activity is 50 times greater than SIR-spheres ${ }^{\circledR}[19]$ and they do not appear to have embolization effects. Thus, Therasphere ${ }^{\circledR}$ do not prevent the administration of following therapies through the same path.

The current protocol for the treatment of HCC includes a first computed tomography (CT) to identify and locate the malignancies. Nowadays, the use of MRI is also increasing thanks to its less invasive nature. Both imaging modalities are performed with contrast agents and at different phases of its distribution within the vasculature. These acquisitions allow a proper evaluation of extent of the tumor, help the radiologist to guide the angiography, and are a baseline reference to assessment of response.

The administration of SIRT requires of course a good knowledge of the arterial tree's branches and bifurcations. For this purpose an arteriography is realized through the injection in the liver arteries of an iodinebased contrast media and the consecutive acquisition of a 3D rotational Cone Beam CT (Fig.1). This acquisition gives a detailed portrait of the arterial tree and allows the interventional radiologist to choose the injection point for future delivery of the treatment. This choice is made by the radiologist without any kind of technological help, making it unreproducible and subject to human error. The hepatic vasculature is very complex and sometimes tortuous, currently it is impossible to have a precise knowledge of it. The choice of this point is currently validated by the injection of a particular kind of proteins, which are likely to imitate the behavior of the microspheres in the blood transport phenomenon. Their distribution is captured by a SPECT-CT acquisition (Single-photon emission computed tomography, coupled to CT). One to two weeks after this diagnostic arteriography, the therapeutic arteriography delivers the microspheres loaded with ${ }^{90} \mathrm{Y}$, and another SPECT-CT is acquired. Efficacy is finally

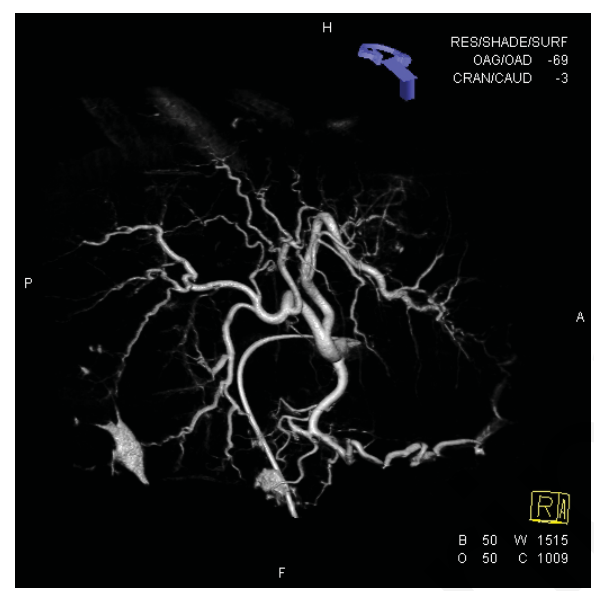

Fig. 1: Example of a 3D Cone-Beam CT angiography. (Centre Eugène Marquis (CEM), Rennes, France)

assessed by morphologic imaging (either MRI or CT) performed every 2 to 3 months.

\section{Methods}

In order to simulate a patient-specific hepatic arterial tree, it is necessary to extract as accurately as possible information from the patient's data. As shown in Fig. 2, we first segment the profile of the liver with tumors from CT at portal phase, whereas hepatic arteries are segmented from 3D Cone Beam CT angiography. After a registration process between the two segmented volumes, we can launch the vascular model previously developed [13, 15].

The computational model described in [13] is able to simulate the liver vascular trees [15] in normal or some pathological situations [18]. This model allows the user to simulate the growth of the hepatic arteries, the portal vein and the hepatic veins, after an initialization phase where the patient specific main vessels can be introduced. The model also requires the contour of the shape of the liver and, if any, the areas where a malignancy is present. Thanks to this information, the model can start growing the smaller vessels. It extends and develops the input vessels, in order to irrigate all the tissue of the liver. When a malignancy is present, the vasculature growth will be denser and more irregular. The necrotic tissue can also be simulated by a decrease of the vascularization until total vanishing of the vessels. The criteria regulating the vessels growth will be given in section 2.1. Information about liver shape, location and dimension of malignancies, as well as the geometry of the main hepatic arteries, can be extracted from the images available in the context of the protocol. This will be presented in section 2.2 . 


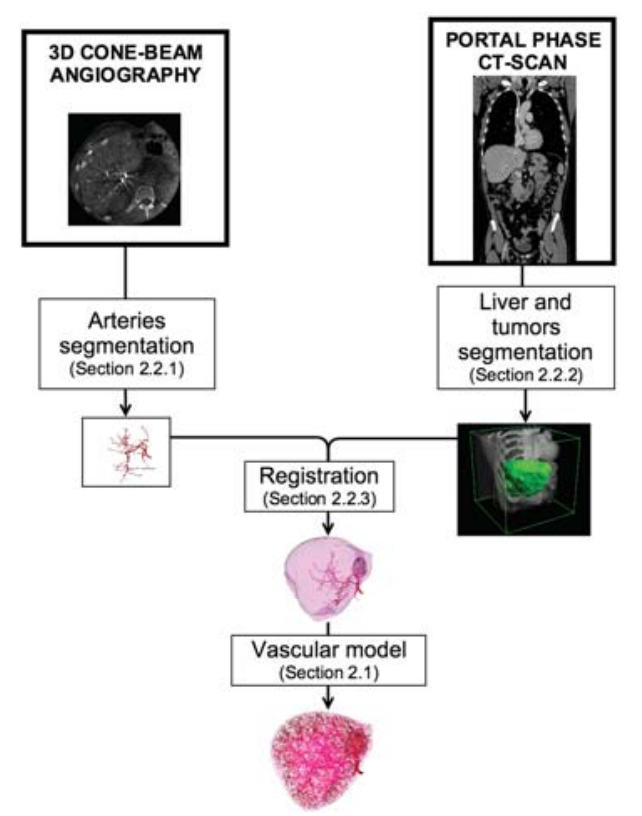

Fig. 2: Outline of the method used to simulate the patientspecific hepatic arterial tree. In order to initialize the model, segmentation of liver with tumors (from portal phase CT) and arteries (from angiography) are needed

\subsection{Vascular model}

The model creates a vascular network that can be made of one or more vascular trees. A vascular tree is composed of vessels that can divide creating bifurcations (Fig. 3a). Each vessel segment (the part of the vessel between two successive bifurcations) is represented as an ideal, rigid tube with fixed radius, wall thickness, length and position. Based on morphometrical investigation [27], it is assumed that the vascular tree is a binary tree.

\subsubsection{Simulation of vascular growth}

The simulation [15] is initialized by a limited vascular network, consisting of a few vessel segments based for example on standard anatomical data or patientspecific segmented images. Further development of vascular trees is modeled as an analogy to a hyperplasia process (an increase in the amount of tissue). The increasing needs of the growing tissue induce the development of the vascular network which is responsible for blood delivery.

Tissue is represented by a set of macro-cells that are regularly (but randomly) distributed inside the predefined 3D shape (Fig. 3b). A macro-cell corresponds to a small part of the tissue, including mainly capillaries, with a predefined average size, defined for healthy macro-cells as $0.125 \mathrm{~cm}^{3}$. The need of each macro-cell

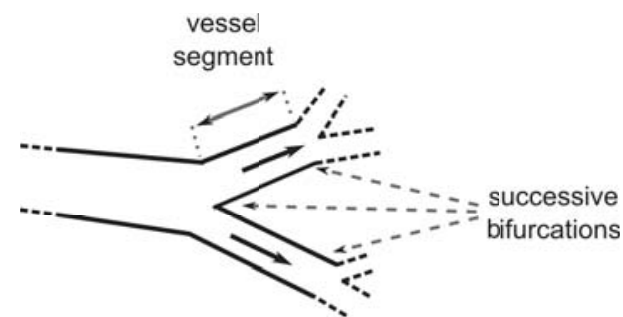

(a) Part of a binary vascular tree

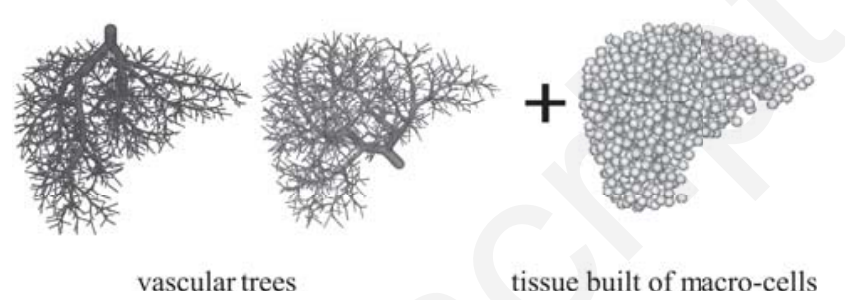

(b) Vascular networks (arteries and veins) and tissue built of macro-cells

Fig. 3: General overview of the model simulating the hepatic vascular trees and tissue

for blood is defined by its class (healthy, cancerous, necrotic, ...), which determines most of its structural and functional properties (e.g. size, birth/death probability), as well as physiological features (e.g. blood pressure, blood flow rate). For example, tumor macro-cells have a higher birth probability than the healthy ones, and their spatial density is higher. Moreover, different classes allow various pathological changes to be investigated. Some of the macro-cell features are described by statistical distributions in order to introduce a natural variability. The geometry of the smallest vessels, i.e. capillaries, is not considered as they are hidden in the macro-cells. As a result, anastomoses (mutual vessel intersections) that occur mainly among vessels with very small radii are not taken into account.

The algorithm of vascular development is divided in cycles. In consecutive cycles, each macro-cell can divide and give birth to a new macro-cell or die (analogy to mitosis and necrosis processes). The algorithm is initialized with an initial set of macro-cells, placed at every end of the initial tree. The newly appeared macrocells are initially not supplied by the existing vascular network. Thus, the procedure of sprouting new vessels towards the new macro-cell is performed. Several candidate bifurcations, constrained by a maximal distance from the macro-cell, are created. Based on them, temporary vascular structures appear. This process can be considered as a kind of competition, since only one vessel (with the lowest sum of volumes, local minimization) is finally chosen as the permanent one. Afterwards, the characteristics of the vessels (i.e. blood flow, pressure 
etc.) are recalculated to fulfill all the used physical constraints.

At every bifurcation, two physical constraints are in effect. The first one deals with the decreasing vessel radii in the vascular tree, creating a relationship between the radius of a father vessel and the radii of its two descendants [14]. Second, the matter conservation law must be observed at each bifurcation: the physical quantities of blood entering and leaving a bifurcation must be identical. Blood flow is modeled in a simplified manner. It is considered as a Newtonian (with constant viscosity $\mu$ ), incompressible fluid, and its flow is governed by the Poiseuille's law, which relates the pressure difference between the two extremities of a vessel segment $(\Delta P)$ to its blood flow rate $(Q)$, length $(l)$ and radius $(r)$ :

$$
\Delta P=Q \frac{8 \mu l}{\pi r^{4}}
$$

The growth of a tumor region always corresponds to an increase of the total hepatic blood flow [8]. The capillary network is denser even inside the lobules, the elementary components of the liver. In the model, tumor macro-cells and vasculature are defined through three principal characteristics. First, blood flow at the entrance of tumor macro-cells was defined as 3 times higher than in the healthy tissue. This ratio is usually considered to be at least 2 in the literature, but can be much higher [2]. Second, the ratio between birth and death probability of tumor macro-cells was higher than in healthy macro-cells. Last, a denser vascular network was induced through an increase of the spatial density of macro-cells to be irrigated. This is done by authorizing a smaller distance between a newly born macro-cell and the already existing ones [15]. In this way, the number of macro-cells in a same region is higher, and the size of macro-cells is thus implicitly reduced.

Since no information about hepatic arterial pressure is available for those patients, pressure value at the entrance of hepatic artery was taken from the literature [7]. Pressure value at macro-cells level was deduced from the diagram relying blood pressure to vessels diameter in human systemic circuit [17]. We consider that pressure values do not depend on the nature of the tissue (tumor or healthy) and on the patient. The initial parameters for the simulation of the vascular network are given in Table 1. At the algorithm initialization, the macro-cells pressure is the one given in Table 1. Such values are conserved for the surviving macro-cells. Moreover, such same value is assigned to every newborn macro-cell. Therefore, at the end of the tree growth, this pressure value is conserved and is constant on the final set of macro-cells. The different
Table 1: Input parameters for the simulation of the hepatic arterial tree. Initial number of macro-cells corresponds to the number of extremities of the segmented arterial tree

\begin{tabular}{lcc}
\hline & Patient 1 & Patient 2 \\
\hline Input pressure & $90 \mathrm{mmHg}$ & $90 \mathrm{mmHg}$ \\
Macro-cells pressure & $10 \mathrm{mmHg}$ & $10 \mathrm{mmHg}$ \\
Initial number of macro-cells & 39 & 19 \\
Depth of initial binary tree & 15 & 20 \\
\hline
\end{tabular}

characteristics of the initial tree for the two patients derive from the arterial tree segmentation step, as will be clarified in Sec. 3.1.

\subsubsection{Simulation of microspheres distribution}

Recent improvements of the model allow more realistic simulation of liver functionalities, in the aim of SIRT modeling. Preliminary simulations of the choice of microspheres best injection point in the arterial tree can be performed. It is temporarily assumed that microspheres flow in every vessel situated downstream from the injection point, proportionally to the blood flow given by Poiseuille's law. Indeed, an intrinsic property of the vascular model is the notion of blood flow, given by Poiseuile's law, in every vessel and in every macrocell of the vascular tree. This property easily allows us to quantify dose distribution in every tissue macro-cell. Namely, starting from any injection point in the tree, at every bifurcation met, the dose will be distributed proportionally to the blood flow of the two child vessels. Therefore we can compute, based on any chosen injection point, the percentage of the injected activity which reached every healthy or tumor macro-cell. Let's now define $N_{h}$ as the total number of macro-cells representing the healthy liver, and $N_{t}$ as the total number of macro-cells representing the tumor. In the same way, we define $\hat{N}_{h}$ as the number of healthy macro-cells reached by the microspheres, and $\hat{N}_{t}$ as the equivalent for the tumor macro-cells. For every macro-cell $i$, we define the injected activity reaching such macro-cell $i$ as:

$$
A_{i}=A \frac{q_{i}}{Q_{p}},
$$

where $A$ is the total injected activity, $q_{i}$ is the flow rate at the entrance of the macro-cell $i$, and $Q_{p}$ is the flow rate at the injection point $p$. Consequently, we can compute the injected activity that reaches all the healthy tissues by:

$$
A_{h}=\sum_{i \in I_{h}} A_{i}
$$


where $I_{h}$ is the index set of healthy macro-cells, and the injected activity reaching the tumor as:

$$
A_{t}=\sum_{i \in I_{t}} A_{i}
$$

where $I_{t}$ is the index set of macro-cells of the tumor. The percentage of the injected activity to the healthy tissues and to the tumor are finally computed as:

$$
\phi_{h}=100 \frac{A_{h}}{A_{h}+A_{t}} \quad \text { and } \quad \phi_{t}=100 \frac{A_{t}}{A_{h}+A_{t}} .
$$

We can also compute the percentages of the healthy and tumor volumes that are irradiated. The percentage of healthy tissue that is reached by the microspheres is given by:

$$
\mathcal{T}_{h}=100 \frac{\sum_{i \in I_{h}} \mathbb{1}_{A_{i}>0}}{N_{h}}
$$

where the indicator function $\mathbb{1}_{A_{i}>0}$ is equal to 1 when the activity $A_{i}$ of the macro-cell $i$ is strictly positive and 0 otherwise. Respectively, the percentage of treated tumor volume is:

$$
\mathcal{T}_{t}=100 \frac{\sum_{i \in I_{t}} \mathbb{1}_{A_{i}>0}}{N_{t}}
$$

In conclusion, we can compute how much of the injected activity reaches how much of the tumor/healthy tissues.

We developed an interactive software that allows the user to navigate through the tree bifurcations, while an interface continuously shows the percentage of the activity distributed in tumor and healthy tissue, $\phi_{h}$ and $\phi_{t}$, as well as the percentages of irradiated volumes $\mathcal{T}_{h}$ and $\mathcal{T}_{t}$.

\subsection{Images processing}

Image analysis for the extraction of patient specific anatomical data can improve all the major steps of the clinical protocol as hepatopulmonary shunt estimation, dosimetry and response evaluation. In this work, we focus on segmentation of hepatic arteries and shape of liver and malignancies, necessary to initialize the vascular model. All procedures performed in this study involving human participants were in accordance with the ethical standards of the institutional research committee and with the 1964 Declaration of Helsinki and its later amendments. Approval by the institutional Medical Ethics Review Board and proper informed consent were obtained.

\subsubsection{Vessels segmentation}

An extended arteries segmentation can be performed on 3D Cone-Beam CT angiography. Thanks to iodinebased contrast agent, the vessels are highly visible, as shown in Fig. 1. Bibliographic study about vessels segmentation showed no algorithm to automatically reconstruct the tree structure from hepatic vascular network [6], [3], [25]. The arteries are then semi-manually segmented by using EndoSize(c) software (Therenva SAS, Rennes, France). Since this software was originally conceived for the aortic trunk, the segmentation can not be completely automatic, the structure of the hepatic arterial tree being more complex. Every vessel of the arterial tree is identified by some critical points manually chosen (Fig. 4). The 3D coordinates of every selected

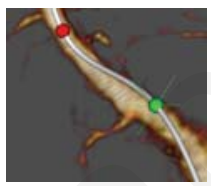

(a) Step 1

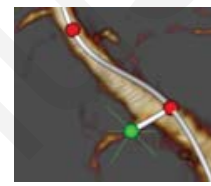

(b) Step 2

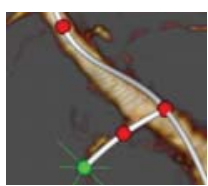

(c) Step 3
Fig. 4: Example of semi-automatic segmentation of vessels with EndoSize. At each step the user defines a new point inside the vessels

point are then automatically saved in a format able to reestablish the binary tree structure of the vessels (tree root, children vessels, tree leaves) needed for the initialization of the simulation in the vascular model. We also measure the diameters of every vessel segment with EndoSize software.

\subsubsection{Liver shape segmentation}

Segmentation of liver and malignancies is performed on full-torso portal phase CT scans by using the MESA software [24], developed at Bialystok University of Technology. MESA is a platform for design and evaluation of segmentation methods and provides tools for management and visualization of medical images. The shape of the liver is extracted with a built-in semi-automatic active surface method [23] that performs a fast fully $3 \mathrm{D}$ segmentation of the volume and requires only small manual corrections. The active surface model is represented as a deformable polygon mesh that evolves under the influence of external image features and internal constraints (Fig. 5). Final results can require some manual corrections, especially near the vessels, performed with a 2D paintbrush-like tool integrated in MESA software. Concerning tumors segmentation, being the ma- 

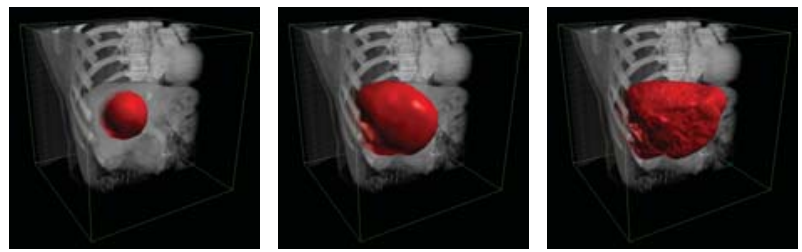

Fig. 5: Surface evolution during four cycles of the liver shape segmentation in MESA software for Patient 2. Segmentation is based on the active surface method [23]

lignancies borders less neat, they are segmented with an active contour model on 2D slices, which requires major manual corrections. The final results are exported to a format readable by the vascular simulation model.

Liver volume is separately measured on a Siemens radiologist workstation, with the vendor supplied application "Volumétrie" in order to validate segmentation.

\subsubsection{D multimodal liver registration}

Unfortunately, there is no available image where satisfactory segmentation of liver shape and vessels can both be realized in a common reference system. In fact, 3D Cone-Beam CT angiography is acquired when contrast agent reaches hepatic arteries, and is still not spread in all the liver tissue (arterial phase). For this reason, liver contour is hardly detectable (Fig. 6a) in 3D angiography. Other images are available, among which two CT acquisitions. The first one at arterial phase (Fig. 6b), when the contrast agent is only in the arteries, and the second one at portal phase (Fig. 6c), i.e. when contrast agent reaches the liver through the portal vein (a few seconds later than through the hepatic artery). Fig. 6b shows that the biggest vessels of hepatic arterial tree are visible on this kind of acquisition (white arrow) and that they can be segmented. However, the best segmentation of liver shape can be achieved on the portal phase volume, when all liver parenchyma is enhanced (Fig. 6c). This leads to the need of a registration process, outlined in Fig. 7, to match information issued from different acquisitions.

Image dimensions are different for every acquisition. Moreover, angiography is acquired one or two weeks after CT. Since liver moves to adapt to body changes, the two images are inevitably different. Furthermore, between the two acquisitions, Patient 1 was subjected to an extra-hepatic artery coil embolization. Namely, a coil was guided to the artery through a catheter and deployed in the chosen vessel with the aim of occluding it. This inevitably induced some artifacts in the resulting Cone-Beam CT (Fig. 6a).

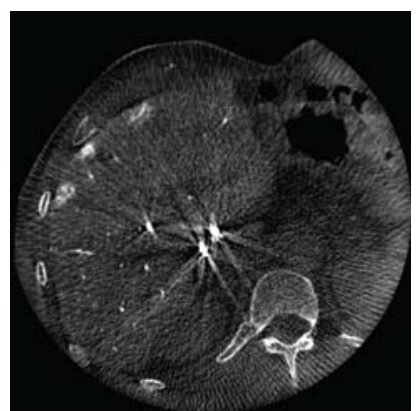

(a) Cone-Beam angiography

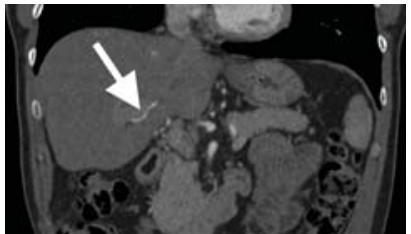

(b) Arterial phase CT

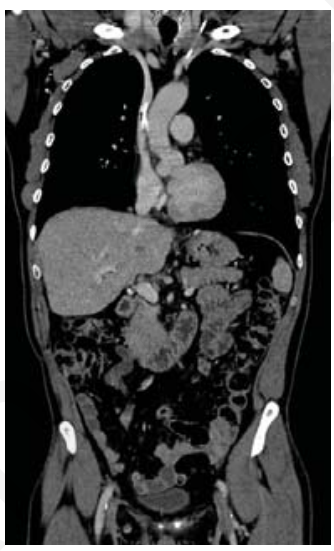

(c) Portal phase CT

Fig. 6: (a) Liver tissue is not enhanced on angiography. Artifacts are due to the presence of metallic coils and surgical clips. (b) White arrow indicate the visible arteries on arterial phase CT. (c) Arteries are not enhanced on portal phase CT. (Centre Eugène Marquis, Rennes, France)

As shown in Fig. 7, the chosen reference volume for registration is portal phase CT. Direct registration from angiography to portal $\mathrm{CT}$ is not possible due to the absence of any similar and common information on the two acquisitions: hepatic arteries almost do not appear on portal CT and liver contour is hardly visible on angiography. This is why we employ arterial phase CT to perform an intermediate step in registration: indeed, this acquisition gives a satisfactory representation of both liver contour and principal hepatic arteries.

Two steps are then identified (Fig. 7): first arterial phase CT is registered on portal phase CT (Fig. 6b to Fig. 6c), next principal hepatic arteries allow registration of angiography on arterial phase CT (Fig. 6a to Fig. 6b). A quick comparison between Fig. $6 \mathrm{~b}$ and Fig. 6c shows that the first step is straightforward. Indeed, images are acquired during the same examination, the difference lying in the acquisition time during contrast agent propagation and in the choice of acquisition parameters. We accomplish this step through a rigid transformation identified automatically by a multimodal registration algorithm based on mutual infor- 


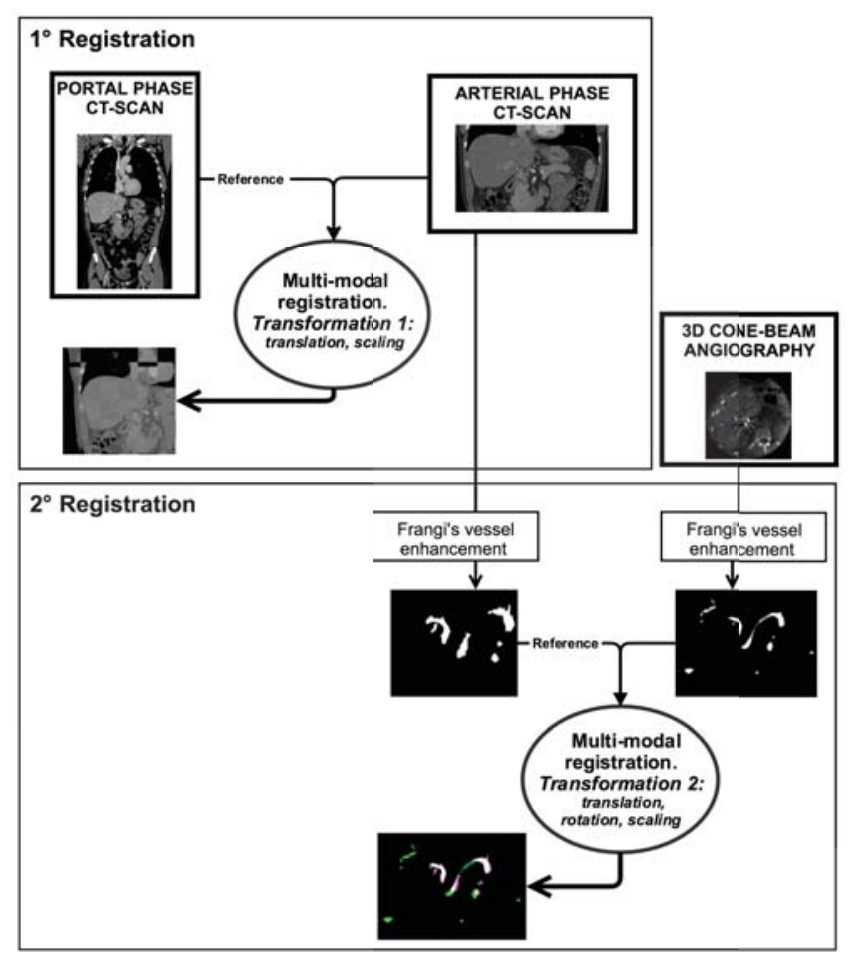

Fig. 7: Registration process between portal phase CT and 3D Cone Beam angiography. In order to initialize the model simulating the arterial tree, registration is needed because liver and tumors are segmented from portal phase CT and arteries are segmented from 3D cone-beam angiography

mation metric [21]. Registration is verified qualitatively through a checkerboard volume.

Registration of angiography on arterial phase CT is based on principal arteries geometry. We draw on both images a region of interest in order to select the biggest hepatic arteries, which are clearly visible even on arterial CT. Vessels enhancement on both arterial phase $\mathrm{CT}$ and angiography is then realized applying a 3D multiscale Frangi's filter [9], [12]. The algorithm uses the eigenvalues $\left(\lambda_{1} \ll \lambda_{2} \ll \lambda_{3}\right)$ of the Hessian matrix of a Mexican hat kernel smoothed version of the image. Such eigenvalues characterize the tubular shape of the vessels and are used to compute a "vesselness function" which is zero if $\lambda_{2}>0$ or $\lambda_{3}>0$, and otherwise is defined as:

$$
\mathcal{V}(s)=\left(1-e^{-\frac{\mathcal{R}_{\mathcal{A}}^{2}}{2 \alpha^{2}}}\right) e^{-\frac{\mathcal{R}_{\mathcal{B}}^{2}}{2 \beta^{2}}}\left(1-e^{-\frac{\mathcal{S}^{2}}{2 c^{2}}}\right),
$$

where $\mathcal{R}_{\mathcal{A}}=\frac{\left|\lambda_{2}\right|}{\left|\lambda_{3}\right|}$ indicates if it is closer to a plate-like or a line structure, $\mathcal{R}_{\mathcal{B}}=\frac{\left|\lambda_{1}\right|}{\sqrt{\left|\lambda_{2} \lambda_{3}\right|}}$ reflects its divergence from a blob-like form, and $\mathcal{S}=\sqrt{\sum_{j=1}^{3} \lambda_{j}^{2}}$ is used to distinguish vessels information from the background. The values of $\alpha, \beta$, and $\mathrm{c}$ have to be tuned by the
Table 2: Frangi's filter parameters for vessel enhancement

\begin{tabular}{l|c|c|c|c|c} 
& Scale range & SR & $\alpha$ & $\beta$ & $\mathrm{c}$ \\
\hline Angiography & {$[1,5]$} & 0.5 & 0.5 & 0.9 & 500 \\
\hline Arterial CT & {$[0.001,2]$} & 0.5 & 0.1 & 0.5 & 10
\end{tabular}

user. The spread of the smoothing kernel corresponds to a scale parameter $s$ which is locally chosen in a given scale range in order to maximize the function $\mathcal{V}$. A scale ratio (SR) gives the step used to go through this range.

Defined parameters are shown in Table 2. We identified a set of parameters convenient for both patients. Next to the Frangi's filter, a grayscale threshold is applied on both images in order to completely remove every non vascular structure. Last, a 3D erosion is applied on angiography in order to simplify the geometry and remove unnecessary information absent on arterial CT. Images are finally registered through an affine transformation (translation, rotation and scaling). The transformation matrix is automatically calculated by a multimodal registration algorithm, based on mutual information metric [21]. The algorithm optimizes images similarity through an iterative evolutionary algorithm [26] for which we identified a set of suitable parameters. Images resolution can be given to the algorithm in order to identify scaling coefficients, ameliorate results and reduce computational time.

Registration is validated through a visual comparison and superimposition of Frangi's enhanced angiography and arterial phase CT. For further validation, we apply the transformation to original angiography and visually compare it to original arterial CT. The identified series of geometrical transformations is finally applied to the list of points describing the arteries, segmented from 3D angiography in EndoSize. Transformation 2 (Fig. 7) registers points to arterial CT, and next transformation 1 is applied to register them to portal $\mathrm{CT}$, where tumors and liver are segmented.

\section{Results}

In order to prepare the initialization of the vascular model, we collect all the needed patients' information in a coherent way. In the present study, we applied the described method to the CT volumes of two patients.

\subsection{Image processing results}

Results concerning arteries segmentation with EndoSize are shown in Fig. 8. The number of leaves of the arterial tree is 39 for Patient 1 and 19 for the other one, 

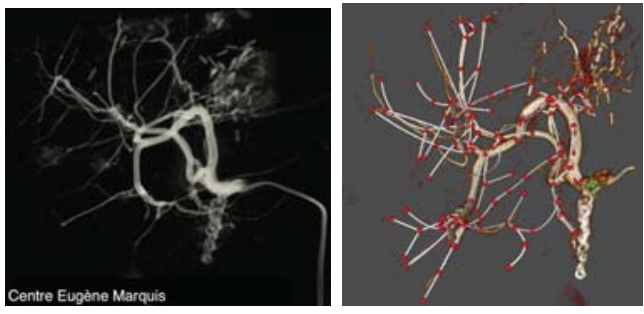

(a) Patient 1
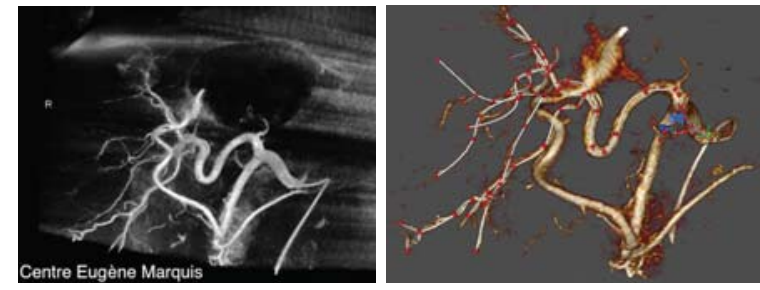

(b) Patient 2

Fig. 8: 3D Cone-Beam angiography (left) and arteries segmentation in EndoSize (right) for both patients. Non-segmented vessels in (b) are extra-hepatic arteries
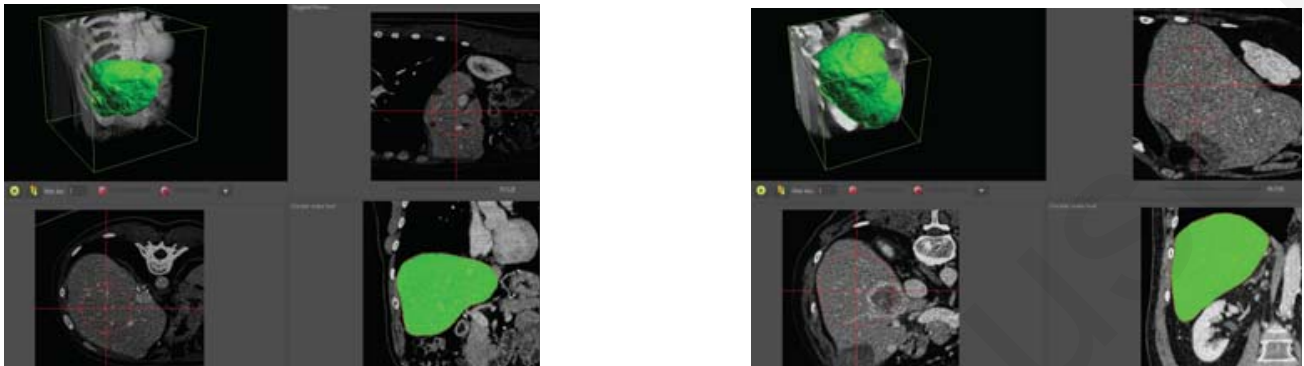

Fig. 9: Result of liver segmentation in MESA: final 3D surface (upper left) and 2D result (lower right) for 2 patients

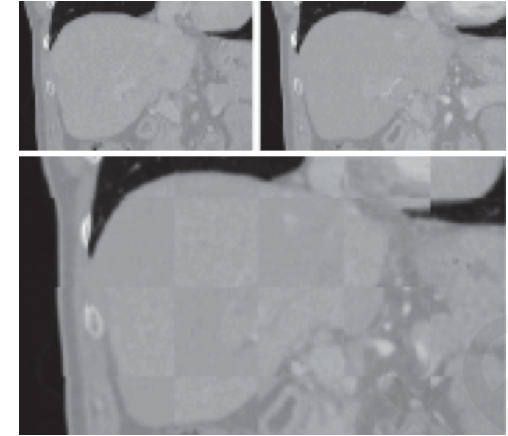

Fig. 10: Registration of arterial CT (right) on portal CT (right). Checkerboard after registration is shown below

as shown in Table 1. Tree depth (i.e. the number of bifurcations present on the longest path from the root to a leave) is 15 for Patient 1 and 20 for Patient 2. The minimum diameter of the segmented arteries is $0.4 \mathrm{~mm}$. The diameter of proper hepatic artery of Patient 1 is $4.8 \mathrm{~mm}$ and $6.5 \mathrm{~mm}$ for Patient 2. We convert automatically this information to the format needed by the vascular model.

Segmentations of liver shapes are performed on an extracted region of interest containing the liver. The total execution time of the deformable surface was less than 10 s on a HP Z420 Workstation. The final mesh consists of about $10 \mathrm{k}$ vertexes and $20 \mathrm{k}$ faces. The segmented liver volume for Patient 1 is equal to $1441 \mathrm{~cm}^{3}$, whereas the volume measured on the Siemens radiolo-

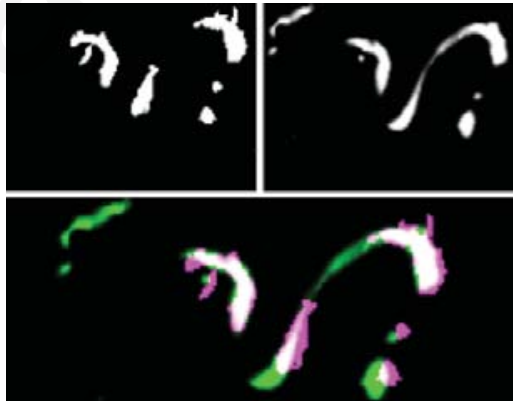

(a)

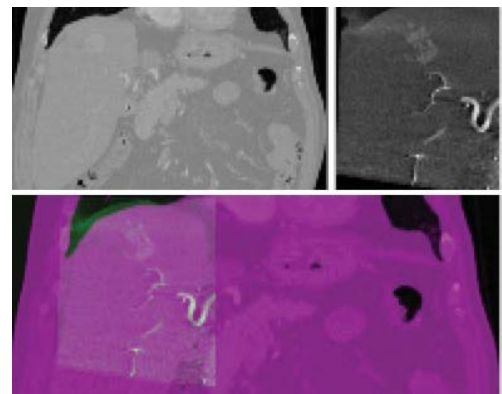

(b)

Fig. 11: (a) Registration of angiography (right) on arterial phase CT (left) is determined from Frangi's enhanced 3D volumes. Superimposition is shown below. (b) The transformation is applied to original angiography (right) and compared to arterial CT (left) for validation. Superimposition is shown below 

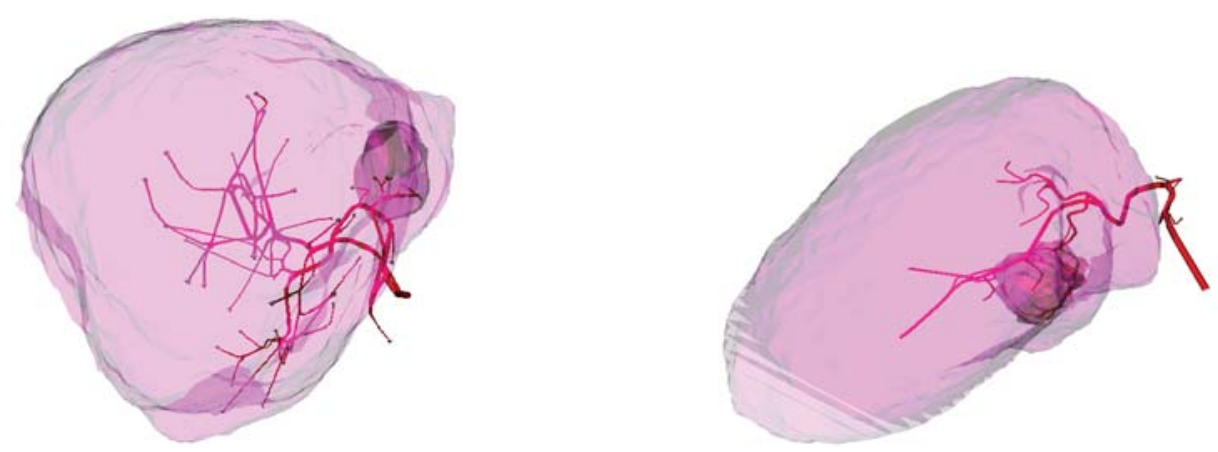

Fig. 12: Registration results between CT and 3D angiography for both patients. Superimposition of liver and tumor contours obtained from portal phase CT and vascular skeleton segmented from angiography
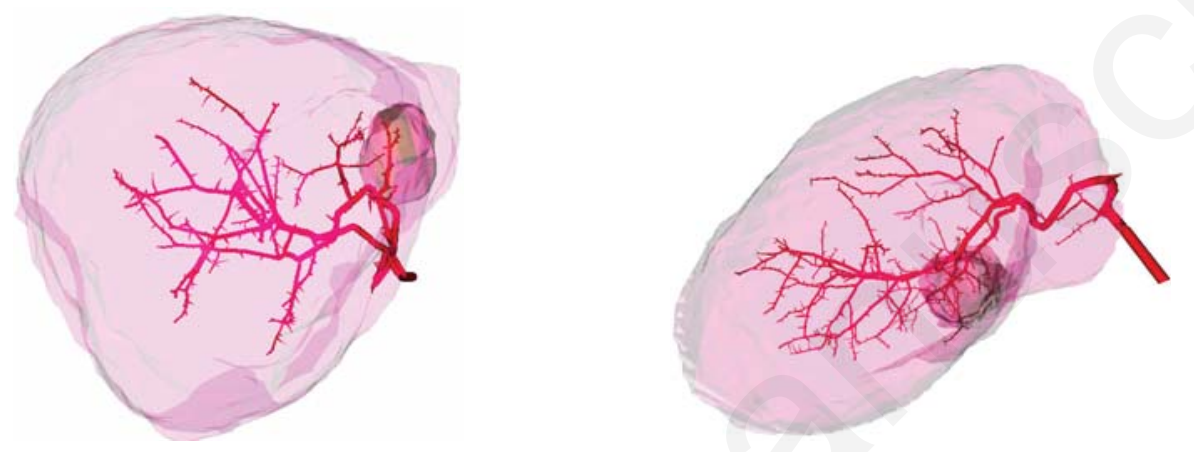

(a)
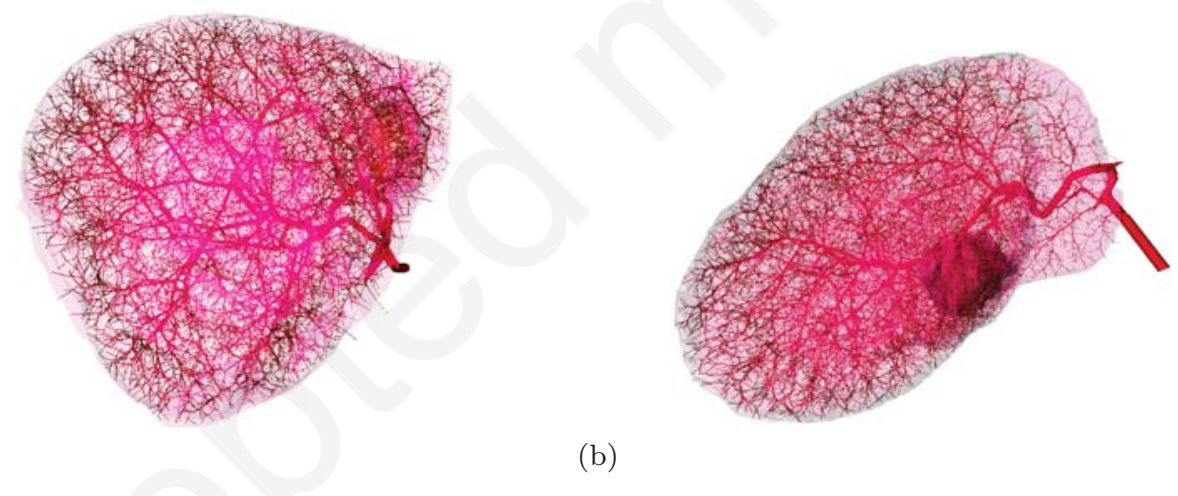

(b)

Fig. 13: Entire simulated hepatic arterial trees for both patients, including tumor vascularization. Only the vessels whose radius is higher than $0.4 \mathrm{~mm}$ are shown in (a)

gist workstation is $1410 \mathrm{~cm}^{3}$. For patient 2, the volume of segmented liver is $2650 \mathrm{~cm}^{3}$, the volume measured by the "Volumétrie" application being $2486 \mathrm{~cm}^{3}$. Segmentation results are shown in Fig. 9.

Registration of arterial phase CT on portal phase CT ( $1^{\circ}$ Registration in Fig. 7 ) is shown in Fig. 10 through a checkerboard volume. Fig. 11a shows the registration of Frangi's enhanced images of angiography to arterial phase CT ( $2^{\circ}$ Registration in Fig.7). The result of registration transformation applied on original angiography is shown in Fig. 11, superimposed to original arterial phase CT. Finally, a 3D visual representation of the input data employed in the vascular model (liver, tumor and arteries) is given in Fig. 12.

\subsection{Vascular model results}

We can then launch the vascular model with the patientspecific data. The model was launched on a portable computer equipped with Intel Core i7 CPU (2.3 GHz) and $16 \mathrm{~GB}$ of RAM under OS X Yosemite. Computational time for the growth of the entire tree is $\sim 10$ minutes, depending on liver size. The radii of the simulated vessels for Patient 1 vary between $1.3 \mathrm{~mm}$ at 


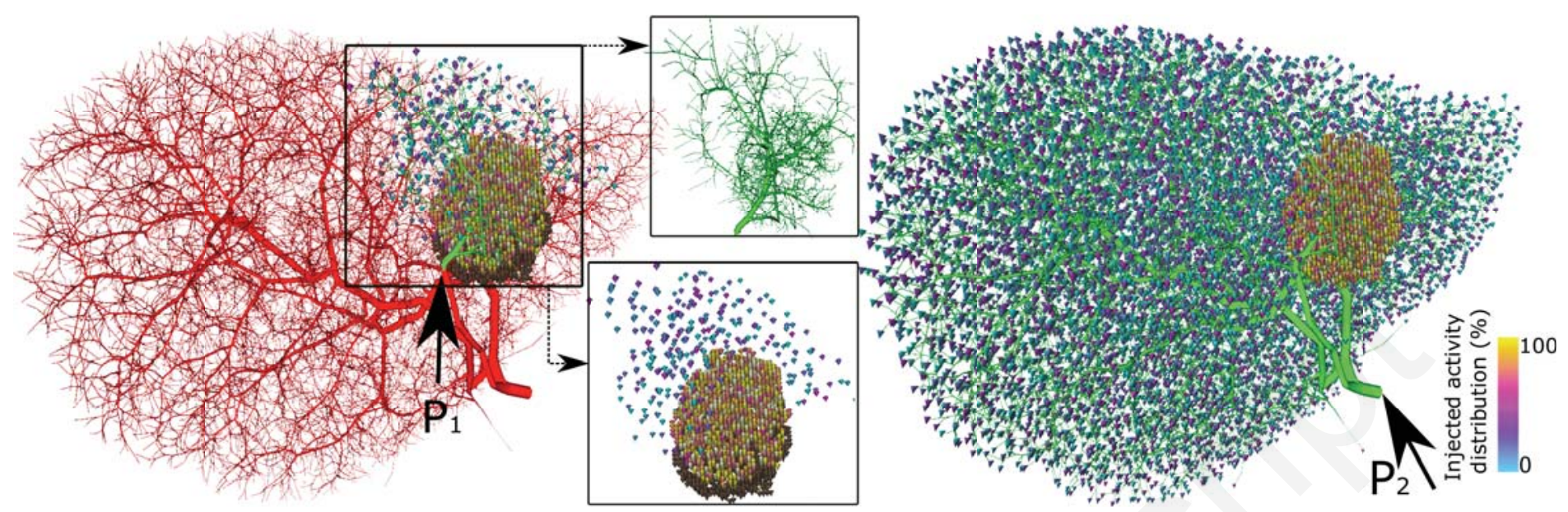

(a) Two different injection points $\left(\mathrm{P}_{1}\right.$ and $\left.\mathrm{P}_{2}\right)$ for Patient 1

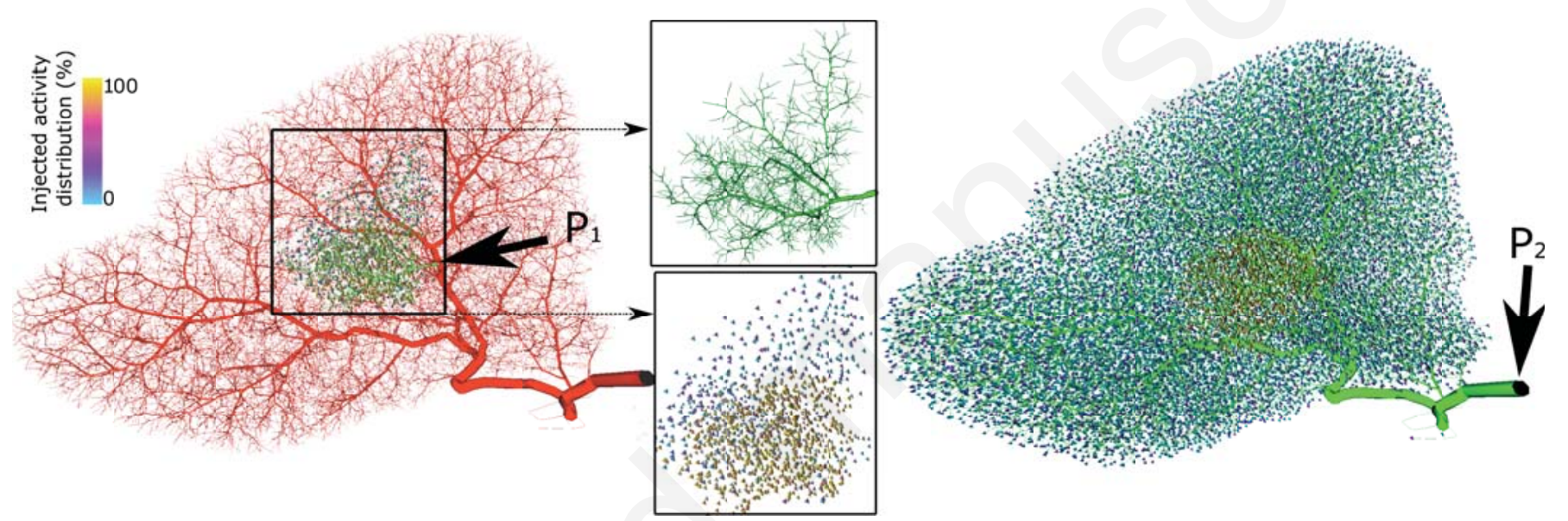

(b) Two different injection points $\left(\mathrm{P}_{1}\right.$ and $\left.\mathrm{P}_{2}\right)$ for Patient 2

Fig. 14: Results of microspheres distribution simulation, based on Poiseuille's law. Light green color marks arteries where microspheres are present, whereas red arteries are not influenced by the treatment. Every macro-cell is visually represented by a triangular shape, colored depending on the radiations intensity: blue-yellow colormap shows the percentage of the administered radioactivity reaching every perfused macro-cell, rescaled by the maximum activity (i.e. $100 A_{i} / \max _{i} A_{i}$ ). Dark green macrocells represent tumor tissue where microspheres are absent. Different injection points are compared for the two patients. (a) In the left image, an optimal catheter tip position $\left(\mathrm{P}_{1}\right)$ is proposed for Patient 1: $71 \%$ of the tumor and $3 \%$ of healthy tissue are injected $\left(\mathcal{T}_{t}=71\right.$ and $\left.\mathcal{T}_{h}=3\right)$. In the right image, catheter tip is positioned at the root of the arterial tree (point $\mathrm{P}_{2}$, for which $\left.\mathcal{T}_{h}=\mathcal{T}_{t}=100\right)$. (b) Similarly for Patient 2 , an optimal catheter tip position $\left(\mathrm{P}_{1}\right)$ is proposed in the left image: $64 \%$ of the tumor and $2 \%$ of healthy tissue are injected $\left(\mathcal{T}_{t}=64\right.$ and $\left.\mathcal{T}_{h}=2\right)$. In the right image, catheter tip is positioned at the root of the arterial tree (point $\mathrm{P}_{2}$, for which $\mathcal{T}_{h}=\mathcal{T}_{t}=100$ )

proper hepatic artery and $0.022 \mathrm{~mm}$ at the smallest arteriole. Concerning Patient 2, the obtained radius of the proper hepatic artery is $2 \mathrm{~mm}$, whereas the smallest radius measures $0.03 \mathrm{~mm}$.

Simulated hepatic arterial trees are shown in Fig. 13. In Fig. 13a only the vessels whose radius is higher than $0.4 \mathrm{~mm}$ are visualized. Comparison to Fig. 12 shows that the original shape of segmented vessels, used for the vascular model initialization, is preserved during the simulation of the arterial tree growth. Tumor vasculature was denser than in the healthy tissue due to the higher authorized density of macro-cells in this region.

\subsection{Application to microspheres distribution in liver}

Since our final aim is to simulate the injection of Theraspheres, we analyze their distribution under simplifying hypotheses, i.e. we simulate microspheres distribution as to be proportional to blood flow given by Poiseuille's law. Under such assumptions we are able to compute, depending on catheter tip placement, the percentage of injected healthy and tumor tissue $\left(\mathcal{T}_{h}\right.$ and $\left.\mathcal{T}_{t}\right)$, as well 
as the percentage of the injected dose which deposits in healthy and tumor liver $\left(\phi_{h}\right.$ and $\left.\phi_{t}\right)$. As shown in Fig. 14, we can propose an optimal injection point specific to every patient. Many patient-specific factors play an important role in the choice of the optimal point. In the current work, optimization refers to a cost function depending only on injected healthy and tumor tissues percentages.

The point suggested in Fig. 14a (left) for Patient 1 induces radiations to $71 \%$ of the tumor $\left(\mathcal{T}_{t}=71\right)$ and to $3 \%$ of the healthy liver $\left(\mathcal{T}_{h}=3\right)$. The optimal catheter tip position proposed for Patient 2 is show in Fig. 14b (left): we calculated that $64 \%$ of the tumor is injected $\left(\mathcal{T}_{t}=64\right)$, together with $2 \%$ of healthy tissue $\left(\mathcal{T}_{h}=2\right)$. Right images of Fig. 14a and Fig. 14b show respectively the distribution of microspheres if the catheter tip is placed at the root of the arterial tree. In this case, all the tissue is irradiated, but a stronger concentration of radiations can be noticed in tumor cells. We quantified that in this scenario $9.2 \%$ of the injected radioactivity deposits in the tumor for Patient $1\left(\phi_{t}=9.2\right)$ and $27.8 \%$ for Patient $2\left(\phi_{t}=27.8\right)$. This preponderance of microspheres in the tumor can be explained by two reasons: tumor macro-cells are more demanding in blood flow, and vascularization is denser within the tumor. Even if we decided to compare only two injection points for one patient, our model allows the user to easily navigate into the vascular tree while calculating in real time the percentages of dose distribution mentioned above. This appears to be a promising approach in quantification of dose distribution in liver, with the aim of injection mode optimization.

\subsection{Validation of microspheres injection simulation}

In order to estimate the validity of the simulation, we considered the SPECT/CT realized on Patient 2 after the diagnostic and therapeutic angiographies. Table 3 shows the percentage $\phi_{t}$ of the injected radioactivity that deposits to the tumor. The table compares the val-

Table 3: Percentage $\phi_{t}$ of the injected activity which deposits to the tumor for both patients

Model computation Clinical measurements

\begin{tabular}{c|c|c} 
& Model computation & Clinical measurements \\
\hline Patient 1 & $9.2 \%$ & $9.2 \%$ \\
\hline Patient 2 & $27.8 \%$ & $34.7 \%$
\end{tabular}

ues given by the simulation to the measurements clinically realized by an experienced nuclear radiologist at "Eugène Marquis Centre (CEM) for the fight against Cancer", Rennes, France, according to the method de-

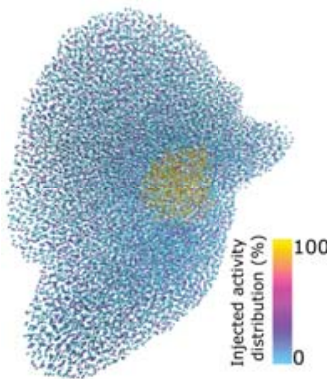

(a) Activity distribution simulation, based on Poiseuille's law. Injected healthy and tumor macro-cells are shown.

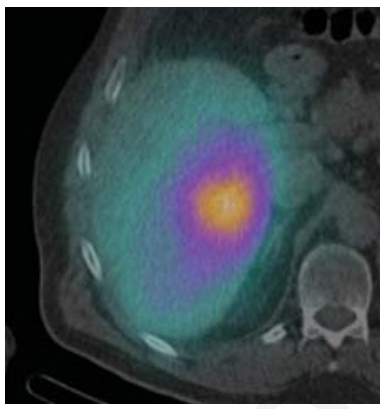

(b) $\mathrm{SPECT} / \mathrm{CT}$ acquisition realized after the ${ }^{90} \mathrm{Y}$ loaded microspheres injection. (CEM, Rennes, France)
Fig. 15: Comparison between simulated and actual microspheres distribution in liver tissue for Patient 2. In this case study, microspheres were injected at the root of arterial tree, therefore $\mathcal{T}_{t}=\mathcal{T}_{h}=100$. Blue-yellow colormap gives the percentage $\phi_{t}$ of microspheres in the injected tissue

scribed in [10]. In both cases and for both patients, catheter tip was placed at the root of the tree, therefore all the healthy and tumor tissues were irradiated $\left(\mathcal{T}_{h}=\mathcal{T}_{t}=100\right)$. The discrepancy concerning the distribution for Patient 2 can be justified by multiple reasons. First, the patient presents several small tumor regions which were not segmented, and therefore not simulated, in this study. Second, the patient underwent a left lobe hepatectomy, which causes radiations deposition in the tissues neighboring the resection. Furthermore, clinical measurements were acquired through the "volumetric analysis" software (Syngo workstation; Siemens), that performs tumors segmentation using a threshold applied to radiations intensity [10]. This can lead to a biased computation of tumor volume, in particular to an overestimation due to extra-tumor radiations deposition.

Fig. 15 shows a comparison between the SPECT/CT and the microspheres distribution simulation for the same patient. It can be observed that in both images microspheres concentration in tumor tissue is higher. This is due to the greater need of blood, characteristic of tumors, and to the dense and tortuous architecture of tumor blood vessels. It should be noticed that, in this case study, radiologist chose to inject at the root of the tree due to the presence of multiple tumors and a cirrhotic tissue, characteristics that were not taken into account in this work. Moreover, a precise comparison of liver shape could not be achieved due to the nature of the two images. Indeed, SPECT/CT is composed of a stack of slices of the hepatic volume, whereas our model offers a $3 \mathrm{D}$ visualization of the volume. 


\section{Discussion}

We can generate a hepatic arterial tree that mimics the contour and function of the patient's vasculature. This process requires segmentation of the shape of liver, tumors, and biggest and more visible hepatic arteries. An important registration process between the two segmented volumes is needed before the vascular model can be initialized. We developed an automatic procedure to register Cone-Beam CT angiography to CT volume. This includes vessels enhancement by Frangi's algorithm. The choice of the Frangi's parameters being relevant, we identified a set of parameters for each modality that appears to be suitable for any patients. In fact, the geometrical characteristics of the vessels (size, brightness) that Frangi's algorithm calculates do not depend on the patient but only on image modality. For the same reasons we can deduce the stability of the parameters identified for the optimization algorithm used for registration.

Initial pressure values at the entrance of the hepatic arteries, as well as more deeply in the arterial tree, needed for the initialization of the vascular model, are determined from literature. In the future we plan to measure it at different levels of the hepatic arterial tree, thanks to a micro catheter.

Results show good correspondence between real and in silico arterial trees. Nevertheless, some differences appear between the segmented arteries used to initialize the model (Fig. 12), and the principal simulated ones (Fig. 13a). This phenomenon shows the importance of finding a compromise between two constraints: first, to preserve the segmented vessels during the simulation; second, the possibility to modify them during the growth of the arterial tree, in order to verify the optimization constraints defined in the model when creating new bifurcations. Future improvements of the model will take into account the first constraint more strictly, as well as the peculiarities of the vasculature due to different pathologies, e.g. vessels tortuosity or arteriovenous shunt. Future work will also concentrate on automatization of hepatic arteries segmentation, and will aim at concrete applicability of this model in actual SIRT protocol.

We are aware that the simulated part of the arterial tree represents the real one only from a statistical point of view. Nevertheless, numerical simulation of microspheres transport and deposition still leads to a statistically realistic microspheres distribution map in the liver tissue, useful in clinical routine. A mean dose distribution map can be estimated by a kernel smoothing of the discrete distribution corresponding to the microspheres centers scatterplot. Moreover, such estimated dose distribution map will be nearly invariant to random changes of microspheres positions, as long as their statistical spatial distribution is preserved.

In order to better take into account liver functionalities, we will focus on the simulation of the blood flow and of microspheres trajectories simulation. It was noted [4] that microspheres transport could not be deduced directly from flow computation. The workflow we proposed here will be improved by recomputing flow and microspheres transport more accurately with computational fluid dynamics (CFD). Two kinds of simulation could be considered: a precise CFD simulation of particles trajectories down to the smallest vessels where a realistic mesh can be defined, and a simplified spheres distribution quantification as the one proposed in this paper.

Simulation of the complete arterial tree has two more interests. First, pressure values given by Poiseuille's law at every level of the simulated tree can be used in order to initialize the pressure boundary conditions necessaries for the CFD simulations. Moreover, it can be used in order to perform a sensitivity study of blood flow, depending on boundary conditions on downstream vessels. Indeed, such conditions are known to be one of the principal difficulties in physiologically based CFD [20]. We plan to deal with complex and large vascular structures, like those presented in this work, that will let us realize a sensitivity study of dose distribution, depending on catheter tip positioning.

\section{Conclusion}

We developed a new procedure for patient-specific hepatic arterial tree modeling, down to arterioles with a diameter of $\sim 0.05 \mathrm{~mm}$. Our model is also able to provide the chaotic and dense vascularization typical of tumors. Simulations are based on the analysis of medical images, from which we segment, and register, patient's hepatic arteries, liver and tumors. We provide a preliminary simulation of microspheres injection and a quantification of their distribution in tumor and healthy tissue, down to small arterioles, based on the Poiseuille's law. The current model is still preliminary and does not differentiate between the behavior of glass microspheres and of the proteins injected during the diagnostic angiography. Nevertheless, it represents an important tool for the initialization of more precise CFD simulations, and it opens the way towards a personalized, quantitative model of SIRT, that will require rigid validations.

Acknowledgements The authors would like to thank Etienne Garin for his contribution to this work. 


\section{References}

1. Andreana L, Isgrò G, Marelli L, Davies N, Yu D, Navalkissoor S, Burroughs AK (2012) Treatment of hepatocellular carcinoma (HCC) by intraarterial infusion of radio-emitter compounds: transarterial radio-embolisation of HCC. Cancer Treat Rev 38(6):641-9, DOI 10.1016/j.ctrv.2011.11.004

2. Andrews JC, Walker-Andrews SC, Juni JE, Warber S, and Ensminger WD. Modulation of liver tumor blood flow with hepatic arterial epinephrine: a spect study. Radiology, 173(3):645-647, 1989.

3. Behrens T, Rohr K, Stiehl HS (2003) Robust segmentation of tubular structures in 3-D medical images by parametric object detection and tracking. Syst Man, Cybern Part B Cybern IEEE Trans 33(4):554-561, DOI 10.1109/TSMCB.2003.814305

4. Childress EM, Kleinstreuer C (2014) Computationally Efficient Particle Release Map Determination for Direct Tumor-Targeting in a Representative Hepatic Artery System. J Biomech Eng 136(1):11,012-11,018, DOI 10.1115/1.4025881

5. Childress EM, Kleinstreuer C, Kennedy AS (2012) A New Catheter for Tumor-Targeting With Radioactive Microspheres in Representative Hepatic Artery Systems-Part II: Solid Tumor-Targeting in a Patient-Inspired Hepatic Artery System. J Biomech Eng 134(5):051,005, DOI 10.1115/1.4006685

6. Esneault S, Lafon C, Dillenseger JL (2010) Liver vessels segmentation using a hybrid geometrical moments/graph cuts method. IEEE Trans Biomed Eng 57(2):276-283

7. Ficher A (1963) The Liver. Elsevier, DOI 10.1016/ B978-1-4832-2824-2.50013-3

8. Folkman J (1971) Tumor angiogenesis: therapeutic implications. N. Engl. J. Med., 285(21):1182-1186

9. Frangi AF, Niessen WJ, Vincken KL, Viergever MA (1998) Multiscale vessel enhancement filtering. Medial Image Comput Comput Assist Invervention 1496:130-137, DOI 10.1016/j.media.2004.08.001

10. Garin E, Lenoir L, Edeline J, Laffont S, Mesbah H, Porée P, Sulpice L, Boudjema K, Mesbah M, Guillygomarc A, Quehen E, Pracht M, Raoul JL, Clement B, Rolland Y, Boucher E (2013) Boosted selective internai radiation therapy with 90Y-loaded glass microspheres (B-SIRT) for hepatocellular carcinoma patients: a new personalized promising concept. Eur J Nucl Med Mol Imaging 20(7):1057-1068

11. GroupHealth (2013) Clinical Review Criteria. SIRT (Selective Internal Radiation Therapy) Therasphere and SIR Sphere for Unresectable Hepatocellular Carcinoma
12. Jimenez-Carretero D, Santos A, Kerkstra S, Rudyanto RD, Ledesma-Carbayo MJ (2013) 3D Frangi-based lung vessel enhancement filter penalizing airways. In: Biomed. Imaging (ISBI), 2013 IEEE 10th Int. Symp., pp 926-929, DOI 10.1109/ ISBI.2013.6556627

13. Jurczuk K, Kretowski M, Eliat PA, Saint-Jalmes H, Bézy-Wendling J (2014) In Silico Modeling of Magnetic Resonance Flow Imaging in Complex Vascular Networks. Med Imaging, IEEE Trans 33(11):2191-2209, URL http://www.ncbi. nlm.nih.gov/pubmed/25020068

14. Kamiya A, Togawa T (1972) Optimal branching structure of the vascular tree. Bull Math Biophys 34(4):431-438, DOI 10.1007/BF02476705

15. Kretowski M, Rolland Y, Bézy-Wendling J, Coatrieux JL (2003) Physiologically based modeling of 3-D vascular networks and CT scan angiography. IEEE Trans Med Imaging 22(2):248-57, URL www.ncbi.nlm.nih.gov/pubmed/12716001

16. Ledzewicz U, Maurer H, Schättler H (2011) Optimal and suboptimal protocols for a mathematical model for tumor anti-angiogenesis in combination with chemotherapy. Math Biosci Eng 8(2):307-323

17. Martini F, Nath JL, Bartholomew EF (2012) Fundamentals of Anatomy and Physiology. Pearson

18. Mescam M, Eliat PA, Fauvel C, de Certaines JD, Bézy-Wendling J (2007) A physiologically based pharmacokinetic model of vascularextravascular exchanges during liver carcinogenesis: application to MRI contrast agents. Contrast Media Mol Imaging 2(5):215-28, DOI 10.1002/ cmmi.147, URL http://www.ncbi.nlm.nih.gov/ pubmed/17874424

19. Murthy R, Nunez R, Szklaruk J, Erwin W, Madoff DC, Gupta S, Ahrar K, Wallace MJ, Cohen A, Coldwell DM, Kennedy AS, Hicks ME (2005) Yttrium-90 microsphere therapy for hepatic malignancy: devices, indications, technical considerations, and potential complications. Radiographics 25 Suppl 1:S41-S55, DOI 10.1148/rg.25si055515

20. Olufsen MS (1998) Modeling of the Arterial System with Reference to an Anesthesia Simulator. PhD thesis, Roskilde University

21. Pluim JPW, Maintz JBA, Viergever MA (2003) Mutual-information-based registration of medical images: a survey. IEEE Trans Med Imaging 22(8):986-1004, DOI 10.1109/TMI.2003.815867

22. Raoul JL, Sangro B, Forner A, Mazzaferro V, Piscaglia F, Bolondi L, Lencioni R (2011) Evolving strategies for the management of intermediatestage hepatocellular carcinoma: available evidence and expert opinion on the use of transarterial 
chemoembolization. Cancer Treat Rev 37(3):21220, DOI 10.1016/j.ctrv.2010.07.006

23. Reska D, Boldak C, Kretowski M (2012) Fast 3D Segmentation of Hepatic Images Combining Region and Boundary Criteria. Image Process Commun 17(4):31-38

24. Reska D, Jurczuk K, Boldak C, Kretowski M (2014) MESA: Complete approach for design and evaluation of segmentation methods using real and simulated tomographic images. Biocybern Biomed Eng 34(3):146-158

25. Smistad E, Elster AC, Lindseth F (2014) GPU accelerated segmentation and centerline extraction of tubular structures from medical images. Int J Comput Assist Radiol Surg 9(4):561-75, DOI 10.1007/ s11548-013-0956-x

26. Styner M, Brechbuhler C, Székely G, Gerig G (2000) Parametric estimate of intensity inhomogeneities applied to MRI. IEEE Trans Med Imaging 19(3)

27. Zamir M, Chee H (1986) Branching characteristics of human coronary arteries. Can J Physiol Pharmacol 64(6):661-668, DOI 10.1139/y86-109

C. Simoncini is currently a Postdoctoral fellow at the Signal and Image Processing Laboratory, University of Rennes 1, INSERM U1099, France, where she received the Ph.D degree in 2017. Her major research interests focus on mathematical modeling in biomedicine, computational fluid dynamics and image processing.

K. Jurczuk received a joined Ph.D. between the University of Rennes 1, France, and the Faculty of Computer Science, Bialystok University of Technology, Poland, in 2013. He is Assistant Professor at the Faculty of Computer Science, Bialystok University of Technology, Poland. His research interests focus on biomedical informatics and parallel computing.

D. Reska is currently a Ph.D. candidate at the Faculty of Computer Science, at Bialystok University of Technology, Poland. His research interests focus on medical image analysis and parallel computing.

S. Esneault is Research Engineer at Therenva SAS, Rennes, France. He received the Ph.D. degree from the University of Rennes 1, France, in 2009. His research interests are in medical image processing.

J.-C. Nunes received the Ph.D degree from the Paris Est University, in 2003. He is Associate Professor at the Signal and Image Processing Laboratory (LTSI), University of Rennes 1, INSERM U1099, France. His major research interests focus on medical image processing (reconstruction, segmentation and registration).
J.-J. Bellanger received the Ph.D. degree from the University of Rennes 1, France, in 1997. He is Professor Emeritus at LTSI, University of Rennes 1, INSERM U1099. His main research interests focus on modeling, identification and statistical signal processing applied to biomedical signals and images.

H. Saint-Jalmes received the Ph.D. degree from the Paris Sud University, Orsay, France, in 1984. He is Professor of biophysics and medical imaging at LTSI, University of Rennes 1, INSERM U1099, France and at Cancer Institute Eugène Marquis, Rennes, France. MRI has been is main research topic for the last 30 years. Besides instrumentation development, including low noise sensors, he is also involved in noninvasive imaging of scattering media.

Y. Rolland received the Ph.D. degree from the University of Rennes 1, France, in 1997. He is Interventional Radiologist and Hospital Practitioner at the Department of Medical Imaging, Cancer Institute Eugène Marquis, Rennes, France. He has been working on liver disease imaging in CT and MRI for 15 years in strong collaboration with academic researchers. He also has a strong experience in radio-embolization of liver tumors with Theraspheres.

P.-A. Eliat received the Ph.D. degree from the University of Rennes 1, France, in 2002. He is Research Engineer at University of Rennes 1 and Engineer at Bio-SCANs PRISM corefacility for the in-vivo small animal MRI development and at the Biosit Federative Research Structure, CNRS UMS 3480, INSERM UMS 018. His main research interests focus on image processing and dynamic MRI modeling for the extraction of quantitative physiological parameters.

J. Bézy-Wendling received the Ph.D. degree from the University of Rennes 1, France, in 1997. She is Associate Professor at LTSI, University of Rennes 1, INSERM U1099. She has been working on medical image analysis and modeling for 15 years in close collaboration with clinicians. Her work focus on image analysis and physiological modeling of tissues and vascular systems with main application to the liver.

M. Kretowski received a joined Ph.D. degree in 2002 from the Faculty of Computer Science, at the Bialystok University of Technology, Poland, and from the University of Rennes 1, France. He is Professor at the Faculty of Computer Science at the Bialystok University of Technology, Poland. His research interests focus on biomedical applications of computer science (modeling for image understanding, image analysis), bioinformatics and data mining. 